SUPPORTING INFORMATION

\title{
Analysis of Temperature-Dependent H/D Exchange Mass Spectrometry Experiments
}

Nastaran N. Tajoddin and Lars Konermann*

Department of Chemistry, The University of Western Ontario, London, Ontario, N6A 5B7, Canada

* To whom correspondence should be addressed. E-mail: konerman@uwo.ca. 


\section{List of SI Items:}

\section{SI Text}

Additional Methods Details (CD Spectroscopy).

Discussion of Global Fitting Strategy.

\section{Supporting Figures}

Figure S1: Thermodynamics of protein unfolding.

Figure S2: Peptic digestion map.

Figure S3: Time- and temperature dependent HDX mass spectra.

Figure $\mathrm{S} 4: k_{c h, i}$ values for $\mathrm{Mb}$.

Figure S5: T-dependence of $k_{c h}$.

Figure S6: Transition state theory applied to opening/closing dynamics.

Figure S7: Segment assignment for global fitting.

Figure S8: Temperature-dependent $\Delta G$ profiles.

SI Appendix: Complete HDX data set with fits. 


\section{SI Methods}

Optical Experiments. Circular dichroism (CD) spectra were recorded on a Jasco J-810 spectropolarimeter (Easton, MD) with a $1 \mathrm{~mm}$ cuvette using $5 \mu \mathrm{M} \mathrm{Mb}$ between $20{ }^{\circ} \mathrm{C}$ and $96{ }^{\circ} \mathrm{C}$. Unfolding profiles were generated by monitoring the CD signal at $222 \mathrm{~nm}$ which is characteristic of $\alpha$-helical secondary structure. ${ }^{1}$ These $\mathrm{CD}_{222}$ profiles were analyzed by using ${ }^{2,3}$

$$
C D_{222}=\frac{\left(y_{N}+m_{N} T\right)+\left(y_{U}+m_{U} T\right) \exp \left(-\Delta G_{g l o b} / R T\right)}{1+\exp \left(-\Delta G_{g l o b} / R T\right)}
$$

where $\left(y_{N}+m_{N} T\right)$ and $\left(y_{U}+m_{U} T\right)$ are the sloped pre- and post-transition baselines, respectively, with $\Delta G_{g l o b}=\Delta H_{g l o b}\left(1-T / T_{m}\right)$.

Discussion of Global Fitting Strategy. Global fitting generally improves the robustness and accuracy of parameters compared to single-curve analyses. At the same time, the number of parameters required for describing the whole data set is reduced. ${ }^{4-8}$ The procedure used in our work involved 22 peptides that were dissected into 44 segments, for a total of $44 \times 3=132$ fitting parameters. One can contrast this to traditional HDX-MS strategies that use expressions such as

$$
\% D=a_{0}+a_{1}\left(1-\exp \left[-k_{a p p \_1} \times t\right]\right)+a_{2}\left(1-\exp \left[-k_{a p p \_} \times t\right]\right)
$$

with five parameters per peptide (or more, when using additional exponentials). ${ }^{9,}{ }^{10}$ For 22 peptides and two temperatures that traditional method would require a minimum of $22 \times 2 \times 5=$ 220 parameters. Here we probed the HDX properties of Mb at eleven (not two) temperatures. In other words, the number of fitting parameters in our global analysis is low, compared to traditionally used approaches. More importantly, the parameters obtained here $\left(\Delta G_{o p U}, \Delta H_{l o c}\right.$, and $\Delta S_{\text {loc }}$ ) directly report on first-principle protein properties. This is in contrast to the $a_{i}$ and $k_{a p p_{-} i}$ values of the equation above, which are difficult to interpret in a structural/thermodynamic context. 

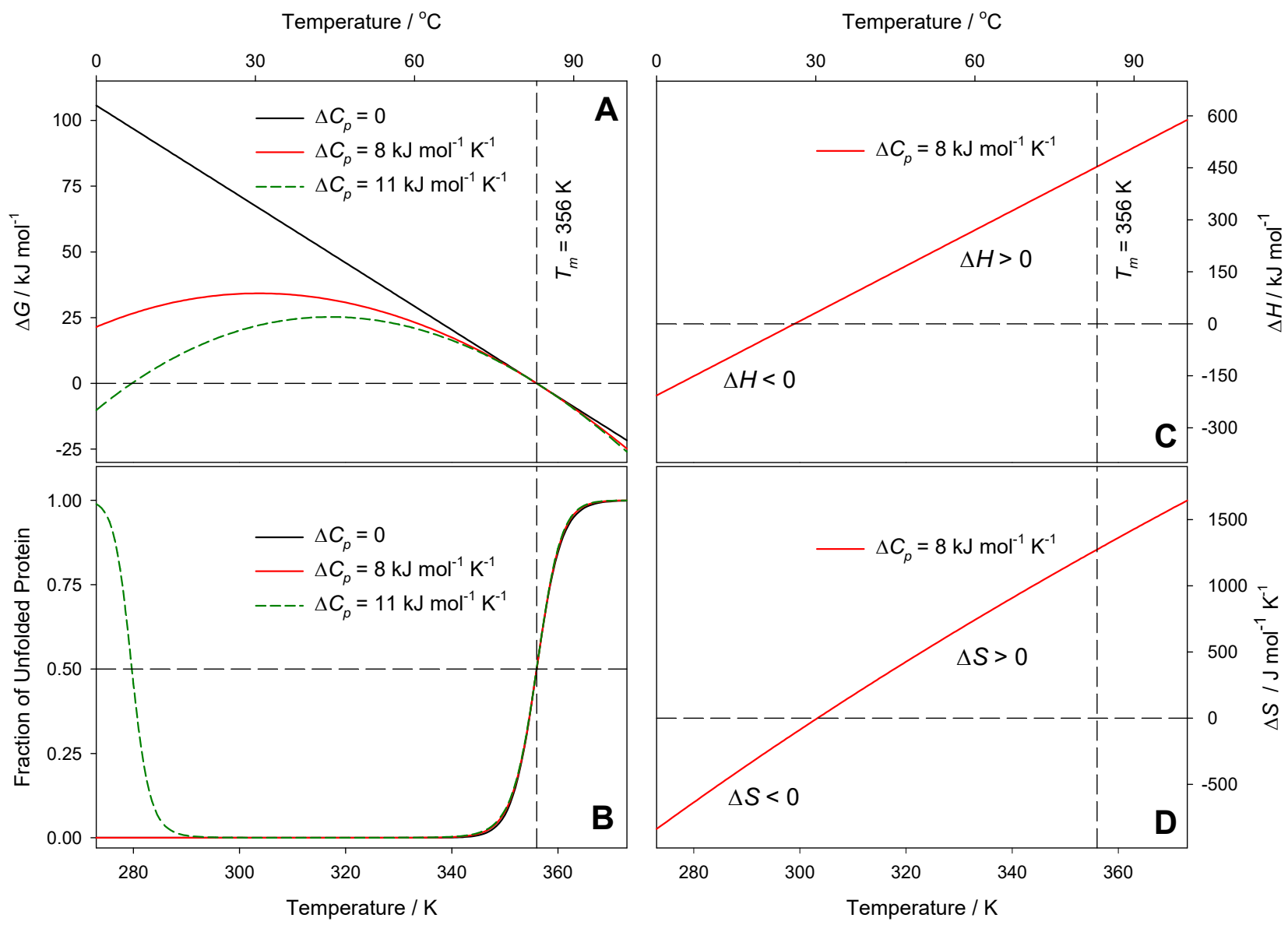

Figure S1. Temperature dependence of a global two-state $\mathrm{N} \leftrightarrow \mathrm{U}$ equilibrium for different values of $\Delta C_{p}{ }^{2,3,11,12}$ (A) Free energy of unfolding; (B) Fraction of unfolded protein. The three data sets share the same $T_{m}=356 \mathrm{~K}$ and $\Delta H\left(T_{m}\right)=453 \mathrm{~kJ} \mathrm{~mol}^{-1}$. The red solid lines apply to $\mathrm{Mb}$ under the conditions of this work $\left(\Delta C_{p}=8 \mathrm{~kJ} \mathrm{~mol} \mathrm{~K}^{-1}\right.$, as in Figure 1). Cold unfolding only occurs for larger $\Delta C_{p}$ values (e.g., $11 \mathrm{~kJ} \mathrm{~mol} \mathrm{~K}{ }^{-1}$, green dashed lines). For temperatures above $\sim 290 \mathrm{~K}$ the three unfolding profiles in (B) are indistinguishable. (C) Enthalpy of unfolding $\Delta H(T)$, and (D) entropy of unfolding $\Delta S(T)$ for $\mathrm{Mb}$ under the conditions of this work.

- Free energy profiles in panel A were calculated from eqs. 3 and 4 (main text) according to $\Delta G(T)=\Delta H(T)-T \times \Delta S(T)$

$$
\begin{aligned}
& =\Delta H\left(T_{m}\right)+\Delta C_{p}\left(T-T_{m}\right)-T\left[\Delta S\left(T_{m}\right)+\Delta C_{p} \ln \left(T / T_{m}\right)\right] \\
& =\Delta H\left(T_{m}\right)-T \Delta S\left(T_{m}\right)+\Delta C_{p}\left(T-T_{m}\right)-T \Delta C_{p} \ln \left(T / T_{m}\right) \\
& =\Delta H\left(T_{m}\right)-T \Delta S\left(T_{m}\right)+\Delta C_{p}\left[\left(T-T_{m}\right)-T \ln \left(T / T_{m}\right)\right] \\
& =\Delta H\left(T_{m}\right)\left(1-T / T_{m}\right)+\Delta C_{p}\left[\left(T-T_{m}\right)-T \ln \left(T / T_{m}\right)\right]
\end{aligned}
$$

In the last line we used $\Delta H\left(T_{m}\right)=T_{m} \Delta S\left(T_{m}\right)$ such that $\Delta S\left(T_{m}\right)=\Delta H\left(T_{m}\right) / T_{m}$.

- Unfolding curves in panel B were calculated using the Boltzmann expression Fraction Unfolded $=\exp (-\Delta G / R T) /[1+\exp (-\Delta G / R T)]$, where $\Delta G=\Delta G(T)$ from panel A.

- On this page we skipped the subscript glob to simplify the notation. 

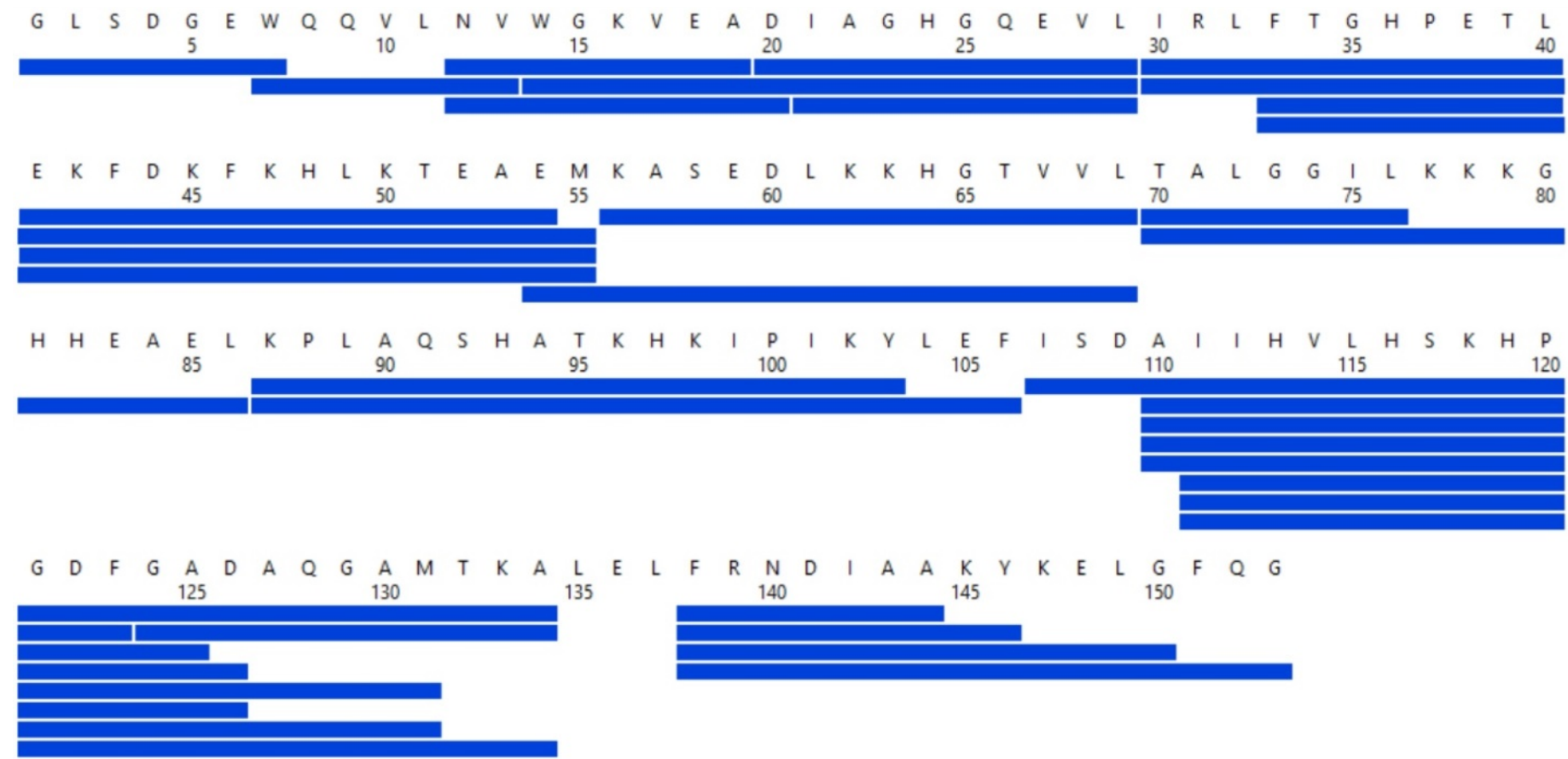

Total: 32 Peptides, $98.0 \%$ Coverage, 3.12 Redundancy

Figure S2. Peptic digestion map, showing the HDX sequence coverage (image generated by Waters DynamX). 

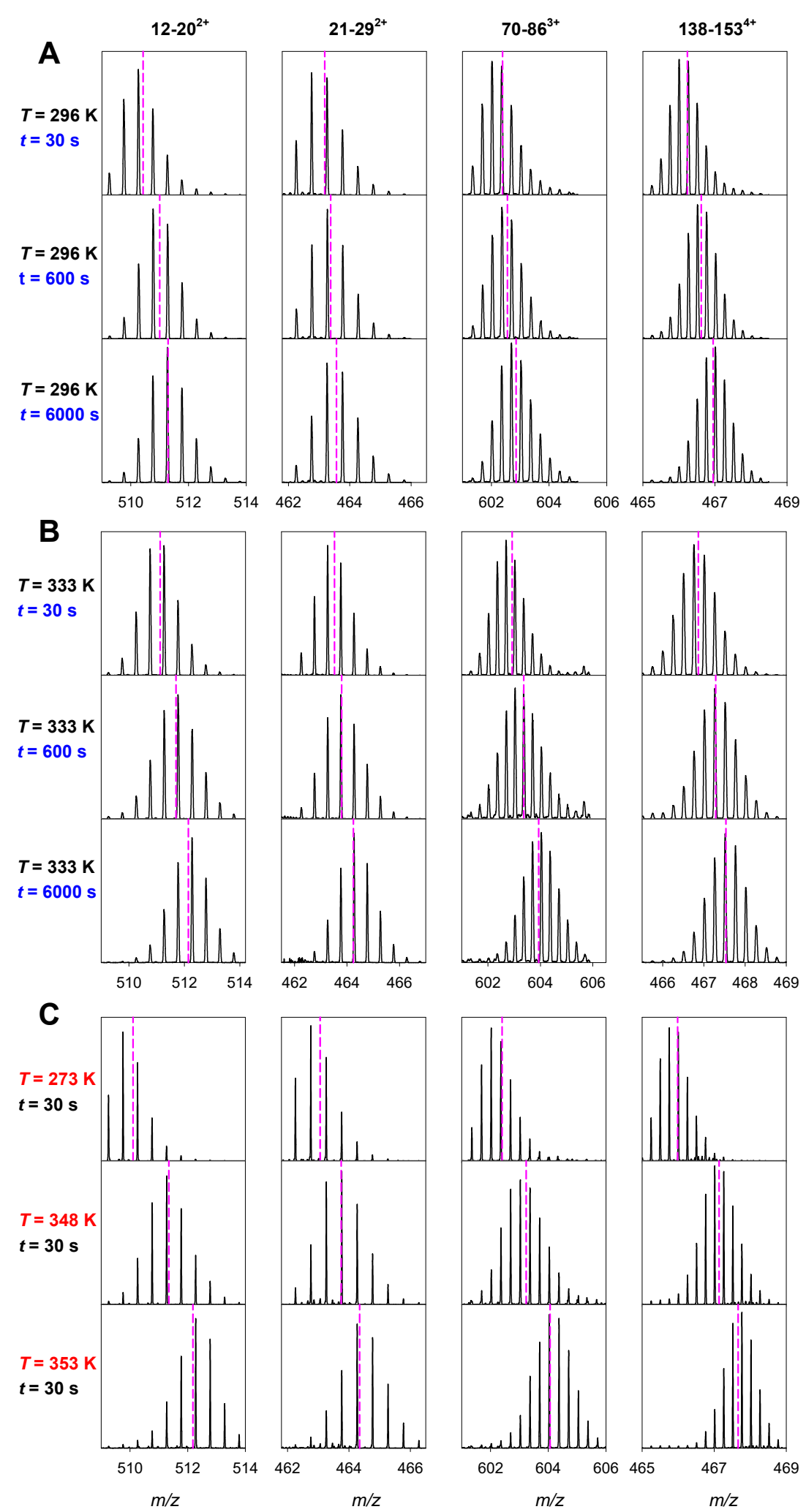

Figure S3. HDX-MS isotope distributions of selected peptic peptides. (A) Data acquired after different labeling time intervals $t$ at a constant temperature of $T=296 \mathrm{~K}\left(23{ }^{\circ} \mathrm{C}\right)$. (B) Same as in panel A, but for $T=333 \mathrm{~K}\left(60{ }^{\circ} \mathrm{C}\right)$. (C) Data acquired at different temperatures $T$ for a constant labeling time of $t=30 \mathrm{~s}$. Vertical dashed lines indicate centroid $\mathrm{m} / \mathrm{z}$ values. 

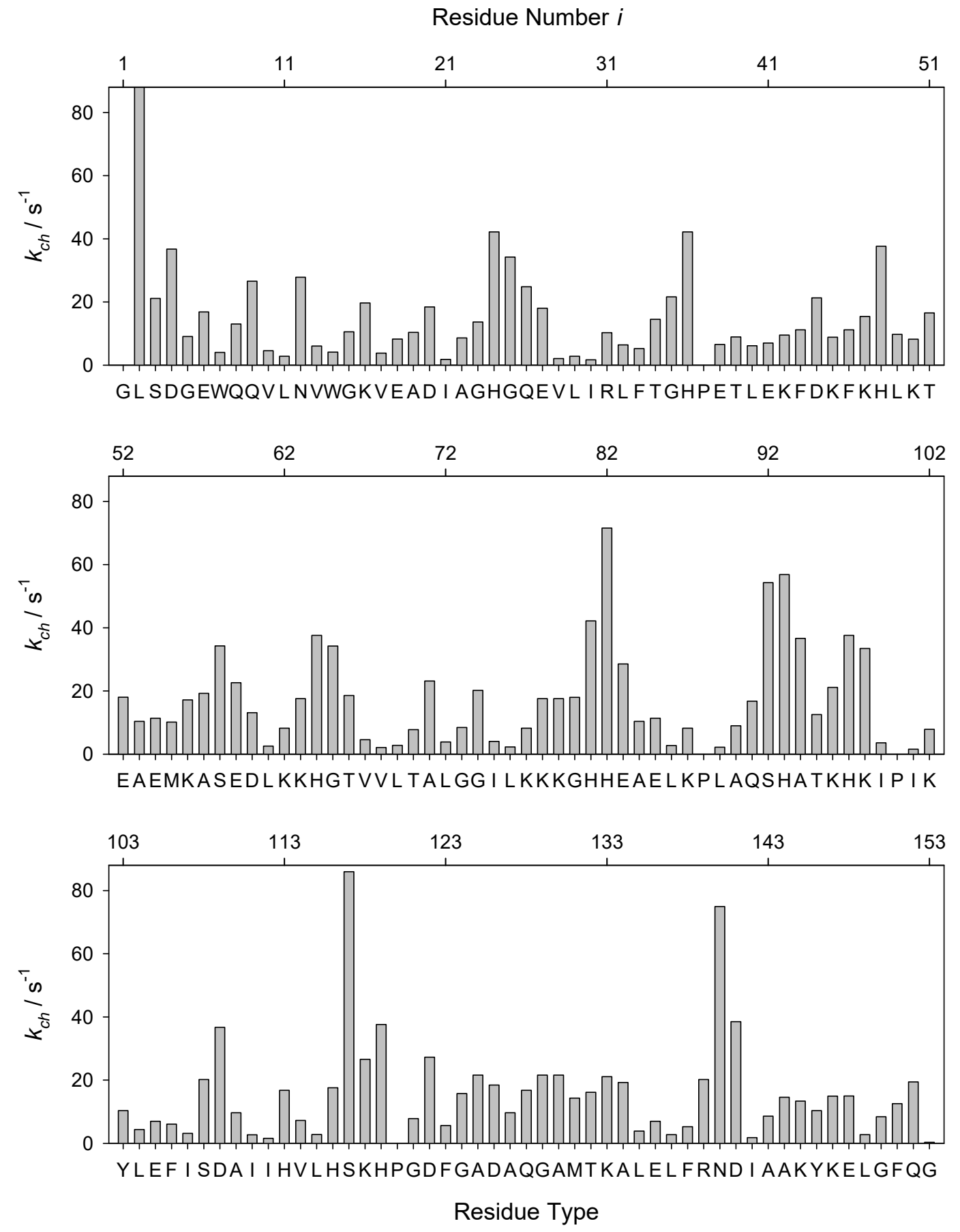

Figure S4. $k_{c h, i}$ values for backbone NH sites along the Mb sequence, for $\mathrm{pD} 7.6$ and $298 \mathrm{~K}$. These values were calculated using Excel files from the Englander Laboratory (http://hx2.med.upenn.edu/download.html). ${ }^{13}$ 


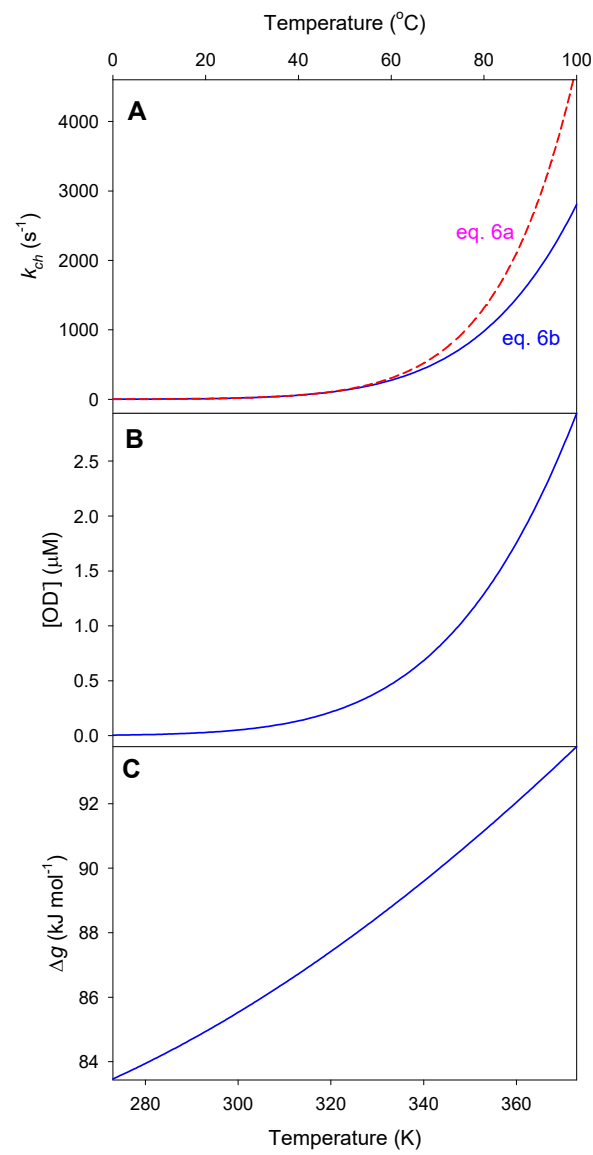

Figure S5. (A) Temperature dependence of $k_{c h}$, calculated using the simple Arrhenius expression of eq. 6a (main text), and by explicitly taking into account how [OD`] changes with $T$ (eq. 6b). All calculations are based on $\mathrm{pD}=$ const. $=7.6$, keeping in mind that the phosphate-buffered solutions used of the current work are stable against $T$-induced changes. ${ }^{14}$ The data shown here are for polyalanine with $k_{c h}(298 \mathrm{~K})=15 \mathrm{~s}^{-1} \cdot 13,15$

(B) Concentration of $\mathrm{OD}^{-}$vs. temperature, calculated as follows:

The ionization constant of $\mathrm{D}_{2} \mathrm{O}$ is $\quad K_{D 2 O}(T)=\left[\mathrm{D}^{+}\right] \times\left[\mathrm{OD}^{-}\right](T)$

such that $\quad \operatorname{pOD}(T)=-\log \left(K_{D 2 O}(T)\right)-\mathrm{pD}$

or $\quad \operatorname{pOD}(T)=-\log (\Delta g(T) / R T)-\mathrm{pD}$

The $\mathrm{OD}^{-}$concentration $(\mathrm{M})$ is thus given by $\left[\mathrm{OD}^{-}\right](T)=10^{-\mathrm{pOD}(T)}$

(C) $\Delta g(T)$ is the free energy change associated with the $\mathrm{D}_{2} \mathrm{O} \leftrightarrow \mathrm{D}^{+}+\mathrm{OD}^{-}$equilibrium. ${ }^{16} \Delta g(T)$ is required for calculating $\left[\mathrm{OD}^{-}\right](T)$.

with the enthalpy

$$
\begin{aligned}
& \Delta g(T)=\Delta h(T)-T \Delta s(T) \\
& \Delta h(T)=\Delta h(298 \mathrm{~K})+\Delta c_{p}(T-298 \mathrm{~K}) \\
& \Delta s(T)=\Delta s(298 \mathrm{~K})+\Delta c_{p} \ln (T / 298 \mathrm{~K})
\end{aligned}
$$

and the entropy

where $\Delta h(298 \mathrm{~K})=59.8 \mathrm{~kJ} \mathrm{~mol}^{-1}, \Delta s(298 \mathrm{~K})=-85.5 \mathrm{~J} \mathrm{~mol}^{-1} \mathrm{~K}^{-1}$, and $\Delta c_{p}=-229.3 \mathrm{~J} \mathrm{~mol}^{-1} \mathrm{~K}^{-1}$. These parameters imply that $k_{B_{-} 298}=3.45 \times 10^{8}$ in eq. $6 \mathrm{~b}$, to ensure that $k_{c h}(298 \mathrm{~K})=15 \mathrm{~s}^{-1}$.

Here we use lower case symbols for solvent-related thermodynamic parameters, whereas upper case symbols in the main text refer to the protein. 
A

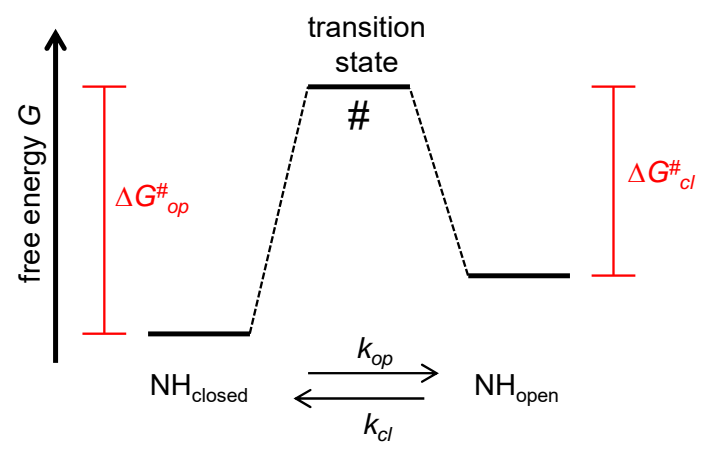

B

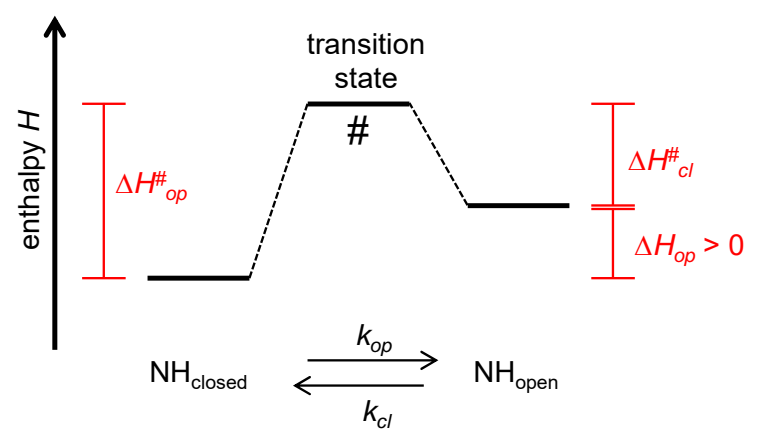

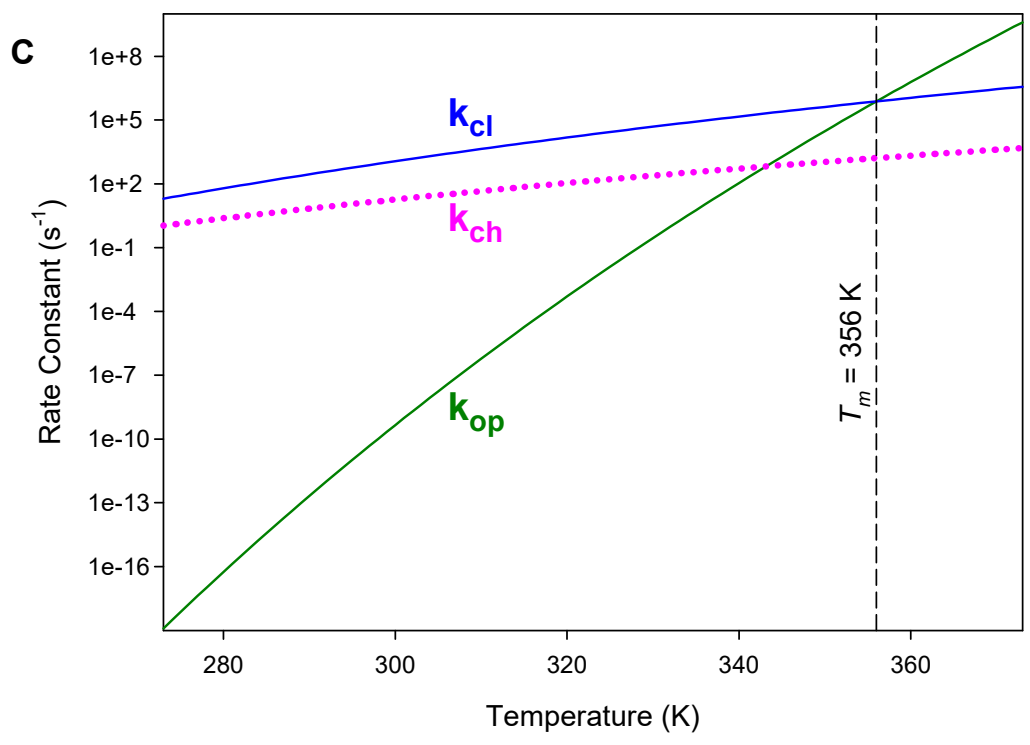

Figure S6: Temperature dependence of backbone amide H-bond opening/closing, and its relationship to the temperature dependence of $k_{c h}$. (A) Transition state theory model of the $\mathrm{NH}_{\text {closed }} \leftrightarrow \mathrm{NH}_{\text {open }}$ equilibrium. ${ }^{2,17} \Delta G^{\#}{ }_{\text {op }}$ and $\Delta G^{\#}{ }_{c l}$ are the activation barrier heights for opening and closing, respectively. The corresponding rate constants are

$$
k_{o p}=\kappa \frac{k T}{h} \exp \left(-\frac{\Delta G_{o p}^{\#}}{R T}\right) \quad k_{c l}=\kappa \frac{k T}{h} \exp \left(-\frac{\Delta G_{c l}^{\#}}{R T}\right)
$$

( $k=$ Boltzmann constant, $h=$ Planck constant, $R=$ gas constant, $T=$ temperature, and $\kappa=$ transmission coefficient). The activation free energies can be dissected into enthalpic and entropic contributions according to $\Delta G^{\#}=\Delta H^{\#}-T \Delta S^{\#}$, such that

$$
k_{o p}=C_{o p} T \exp \left(-\frac{\Delta H_{o p}^{\#}}{R T}\right) \quad k_{c l}=C_{c l} T \exp \left(-\frac{\Delta H_{c l}^{\#}}{R T}\right)
$$

with $\quad C_{o p}=\kappa \frac{k_{B}}{h} \exp \left(\frac{\Delta S_{o p}^{\#}}{R}\right) \quad C_{c l}=\kappa \frac{k_{B}}{h} \exp \left(\frac{\Delta S_{c l}^{\#}}{R}\right)$ 


\section{Figure S6 Caption (continued):}

When expressed in this way, it becomes clear that the temperature dependence of $k_{o p}$ and $k_{c l}$ is governed by the activation enthalpies $\Delta H^{\#}$ op and $\Delta H_{c l}^{\#}$, while the entropy terms can be incorporated into the $T$-independent prefactor. Heat is required to dissociate $H$-bonds $\left(\Delta H_{o p}>0\right)$. This implies $\Delta H_{o p}^{\#}>\Delta H_{c l}^{\#}$ as illustrated in (B), causing $k_{o p}$ to depend more strongly on temperature than $k_{c l}$.

Panel (C) illustrates how $k_{o p}$ and $k_{c l}$ change with temperature. The numerical parameters were chosen to resemble the global $\mathrm{Mb}$ unfolding data of Figure 1, i.e., $\Delta H_{o p}=453 \mathrm{~kJ} \mathrm{~mol}^{-1}$ and $\Delta H^{\#}{ }_{c l}=$ $100 \mathrm{~kJ} \mathrm{~mol}^{-1}$ (estimated from literature data ${ }^{18,19}$ ) such that $\Delta H^{\#}{ }_{o p}=553 \mathrm{~kJ} \mathrm{~mol}^{-1} . C_{c l}$ was arbitrarily chosen as $10^{18} \mathrm{~s}^{-1} \mathrm{~K}^{-1}$ to ensure EX2 conditions with $k_{c l}=20 \mathrm{~s}^{-1}$ at $273 \mathrm{~K}$. This determines the value of $C_{o p}=3 \times 10^{84} \mathrm{~s}^{-1} \mathrm{~K}^{-1}$ to ensure that $k_{o p}=k_{c l}$ at $T_{m}=356 \mathrm{~K}$.

Also included in (C) is a temperature-dependent $k_{c h}$ profile, calculated using the Arrhenius parameters of ref. ${ }^{15}$ for poly-alanine at $\mathrm{pD}=7.6$ (eq. $7 \mathrm{a}$ ).

Key conclusion from the data presented in this Figure: A protein that exhibits EX2 behavior ( $\boldsymbol{k}_{\boldsymbol{c}}$ $>>k_{c h}$ ) at low temperature is likely to remain in the EX2 regime when the temperature is raised. This is illustrated by in panel $(\mathrm{C})$, where $k_{c l}$ remains at least two orders of magnitude above $k_{c h}$ throughout the entire range from $273 \mathrm{~K}$ to $373 \mathrm{~K}$. 


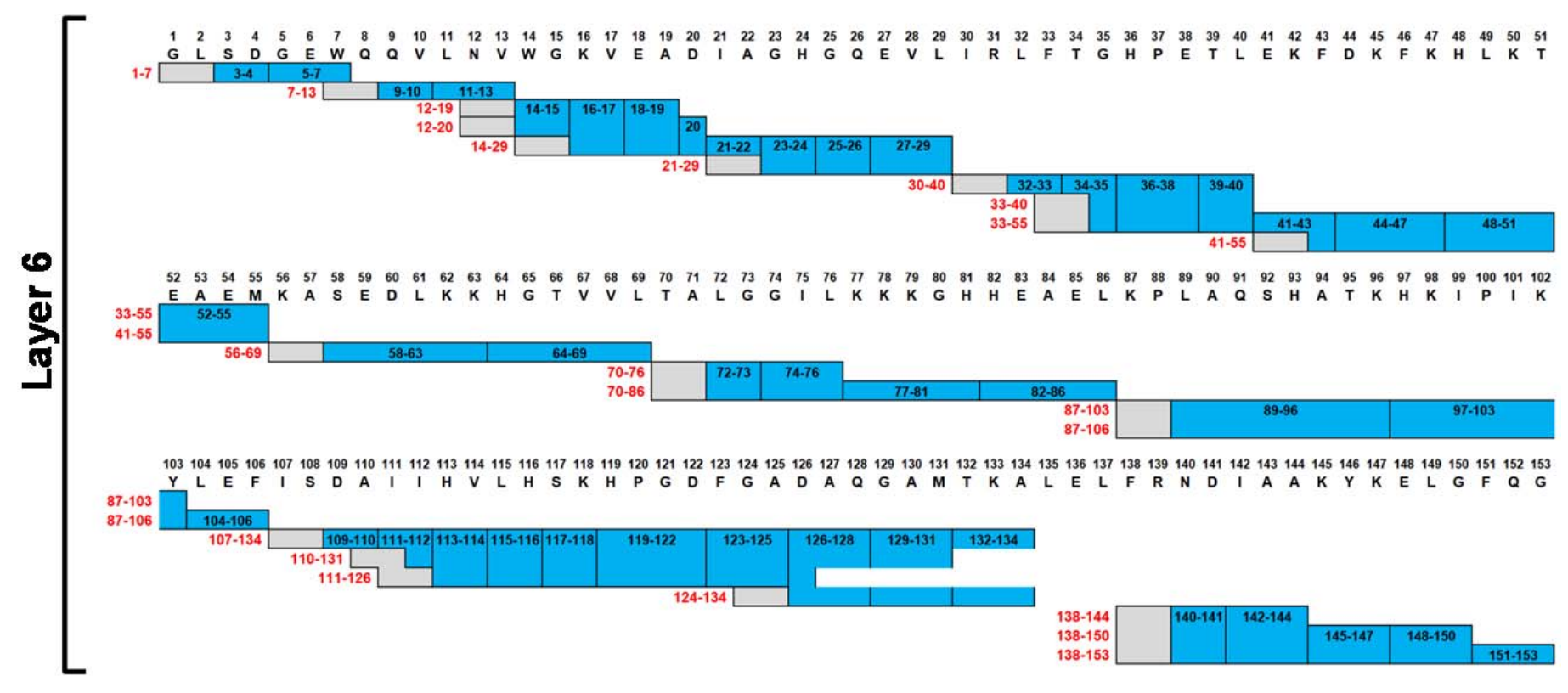

Figure S7. Overlapping peptides (red) used for global fitting, illustrating Layer 6 of the modeling strategy developed here. Blue vertical segments share the same $\Delta G_{o p U}, \Delta H_{\text {loc }}$, and $\Delta S_{\text {loc }}$ across different peptides; the residue range for each segment is indicated. The first two residues of each peptide (gray) were not considered due to back exchange. Preliminary segment boundaries were first determined by analyzing one peptide at a time. If treating a peptide as a single segment did not yield an acceptable fit, it was divided into two, then three segments, etc. For global fitting these preliminary boundaries had to be slightly adjusted to ensure consistency across overlapping peptides (vertical lines in the figure above). 


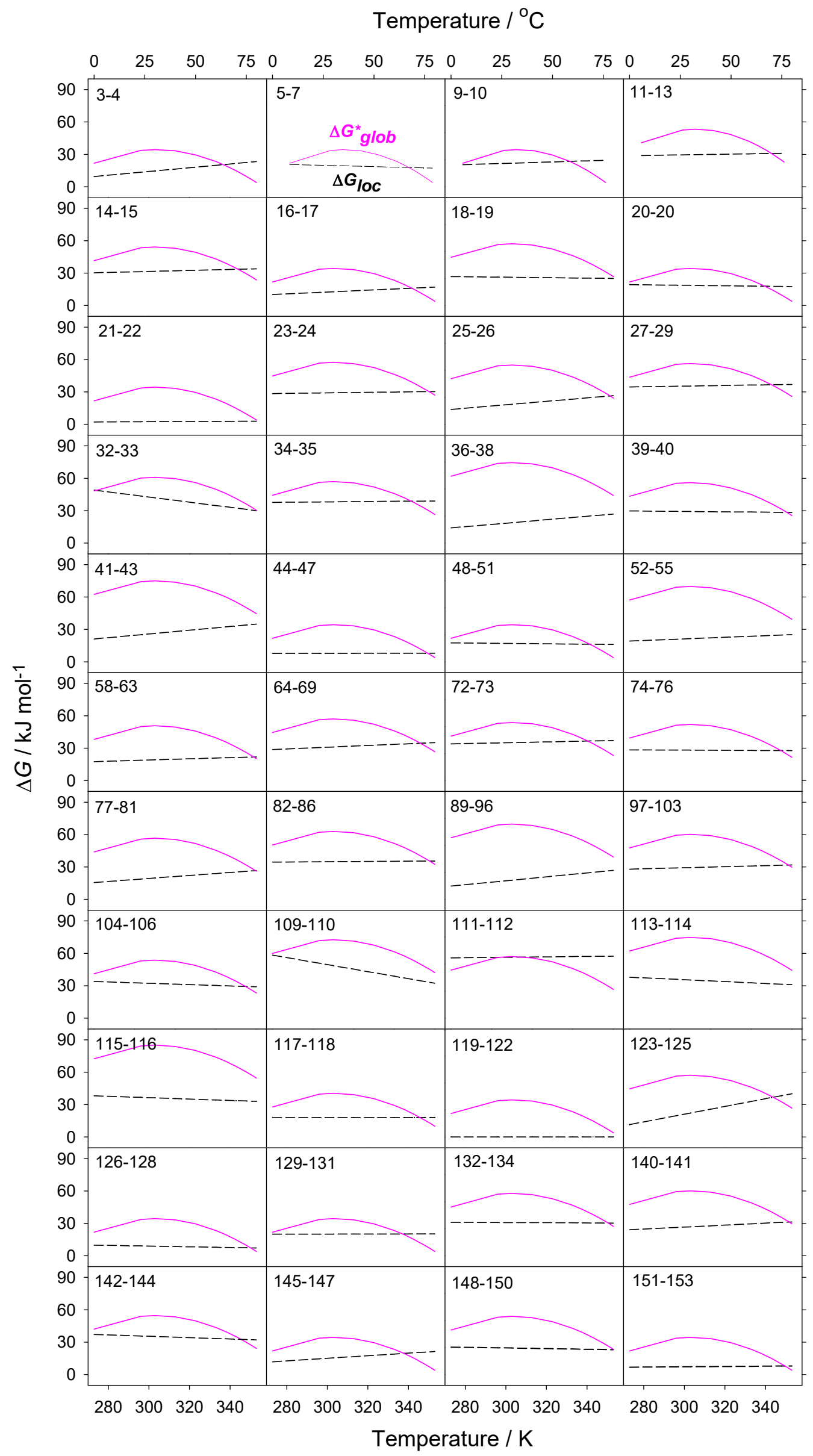

Figure S8.

Complete set of temperaturedependent $\Delta G$ profiles calculated from the $\Delta G_{o p U}$, $\Delta H_{\text {loc }}$, and $\Delta S_{\text {loc }}$ parameters of Figure 4 (main text) . $\left[\Delta G_{\text {glob }}^{*}=\Delta G_{\text {glob }}+\right.$ $\left.\Delta G_{o p U}\right]$ profiles are shown as pink solid lines, $\Delta G_{\text {loc }}$ profiles are shown as black dashed lines for 44 segments along the $\mathrm{Mb}$ sequence. The residues corresponding to each segment are indicated in the individual panels. 
SI Appendix (continued on the following pages): Complete experimental HDX-MS data set (colored dots), with fits (black lines) based on eq. 9 obtained by global analysis of overlapping peptides.
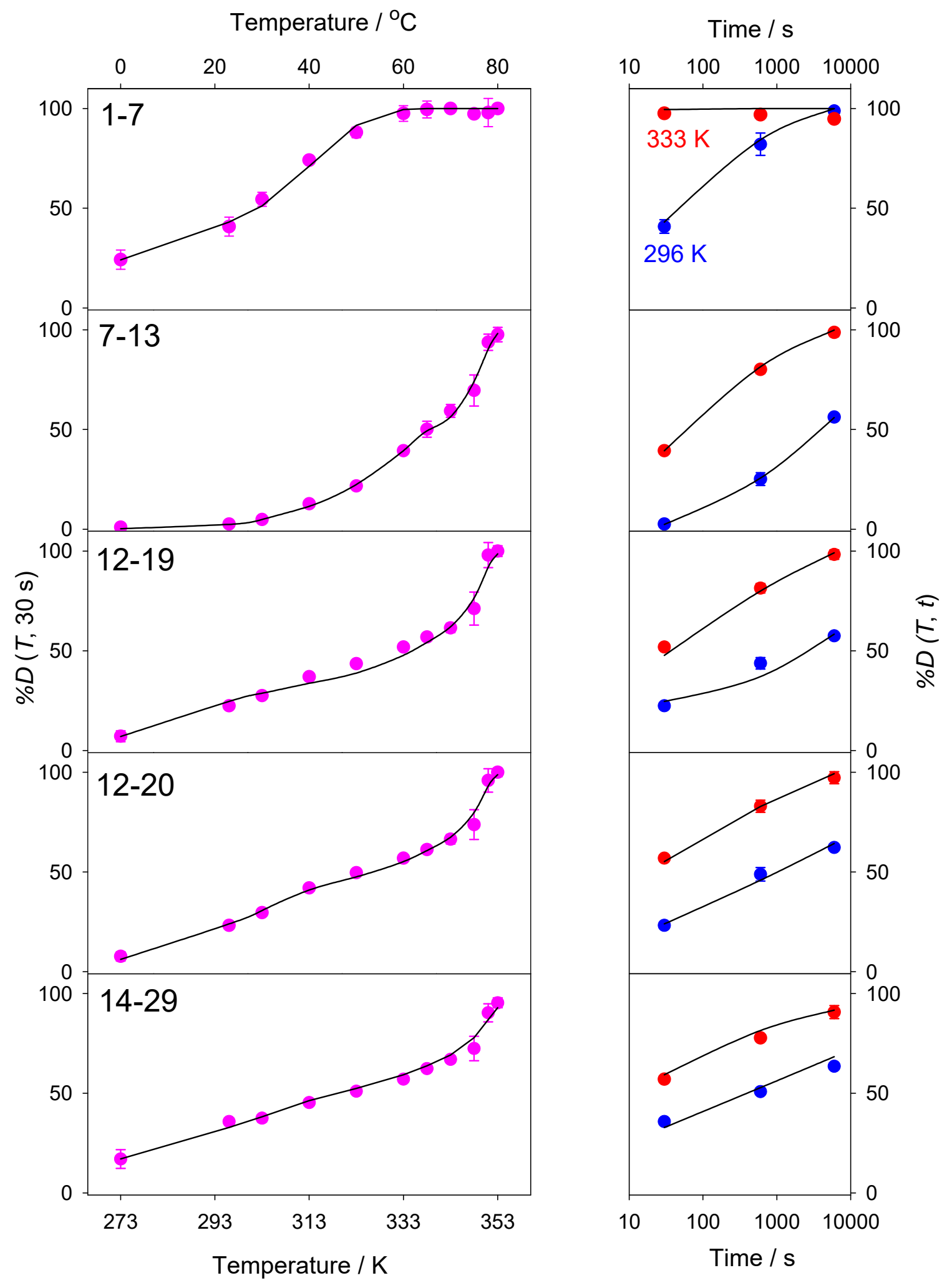


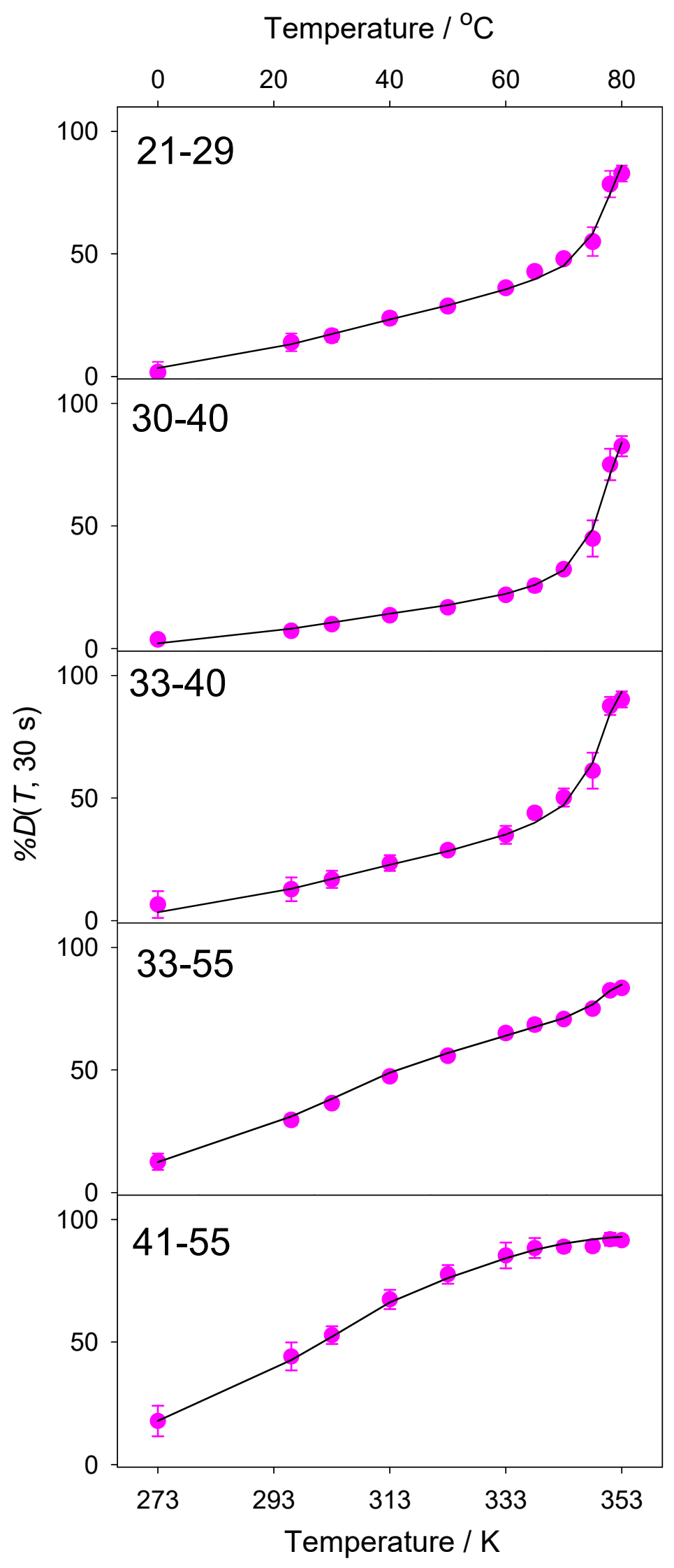

Time / s

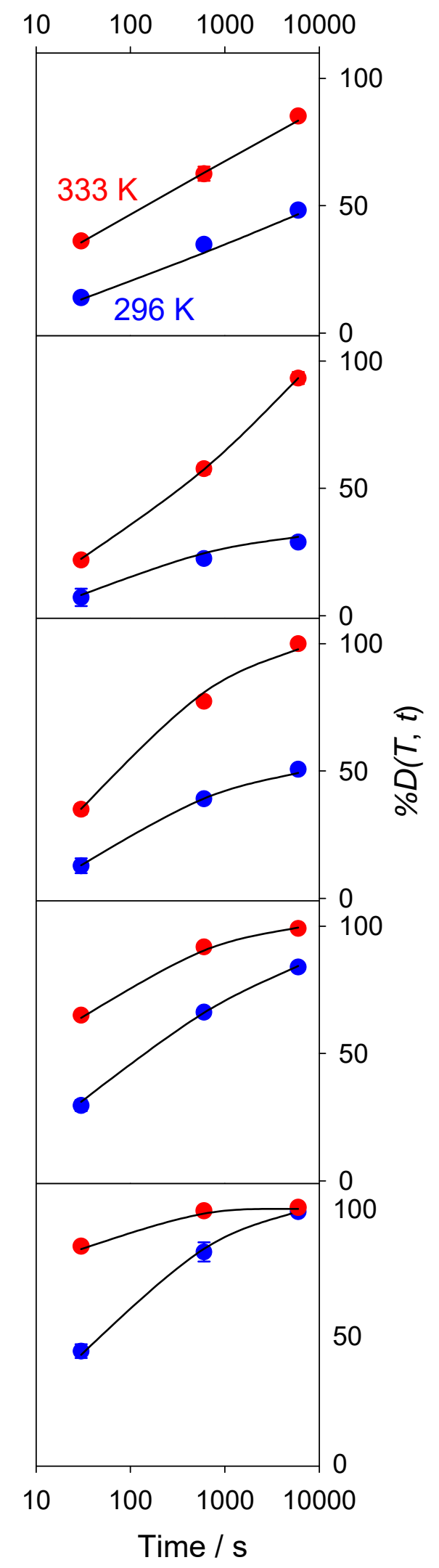




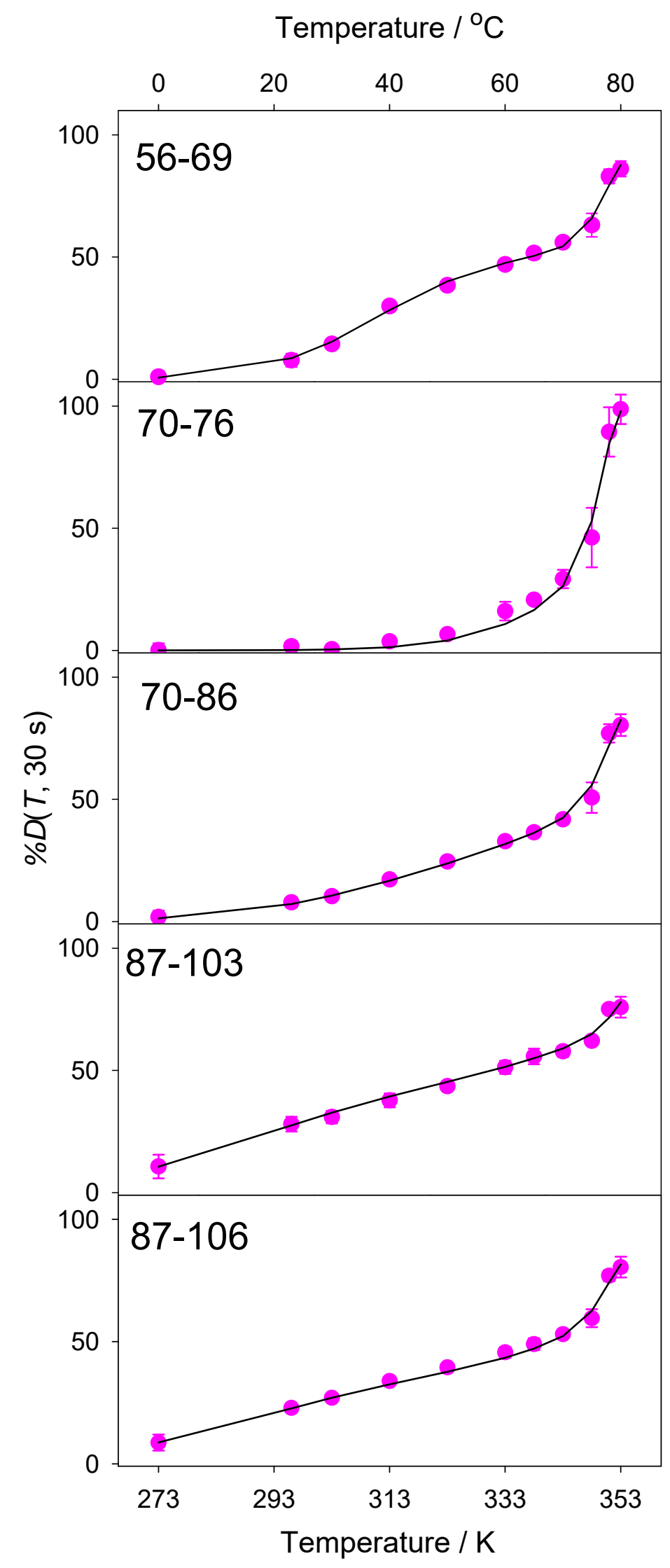

Time / s

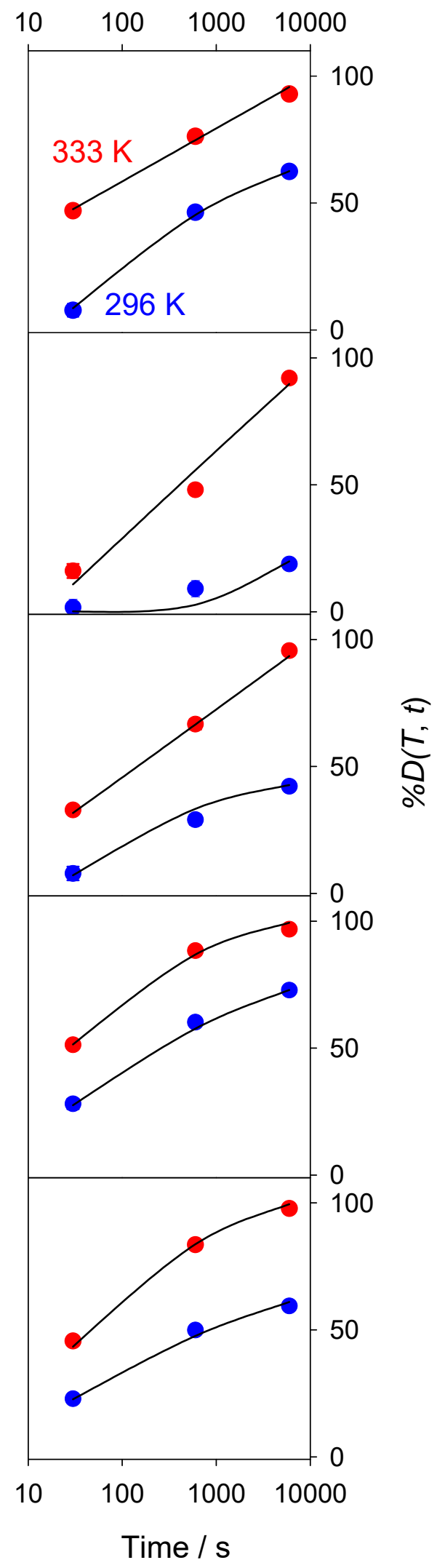




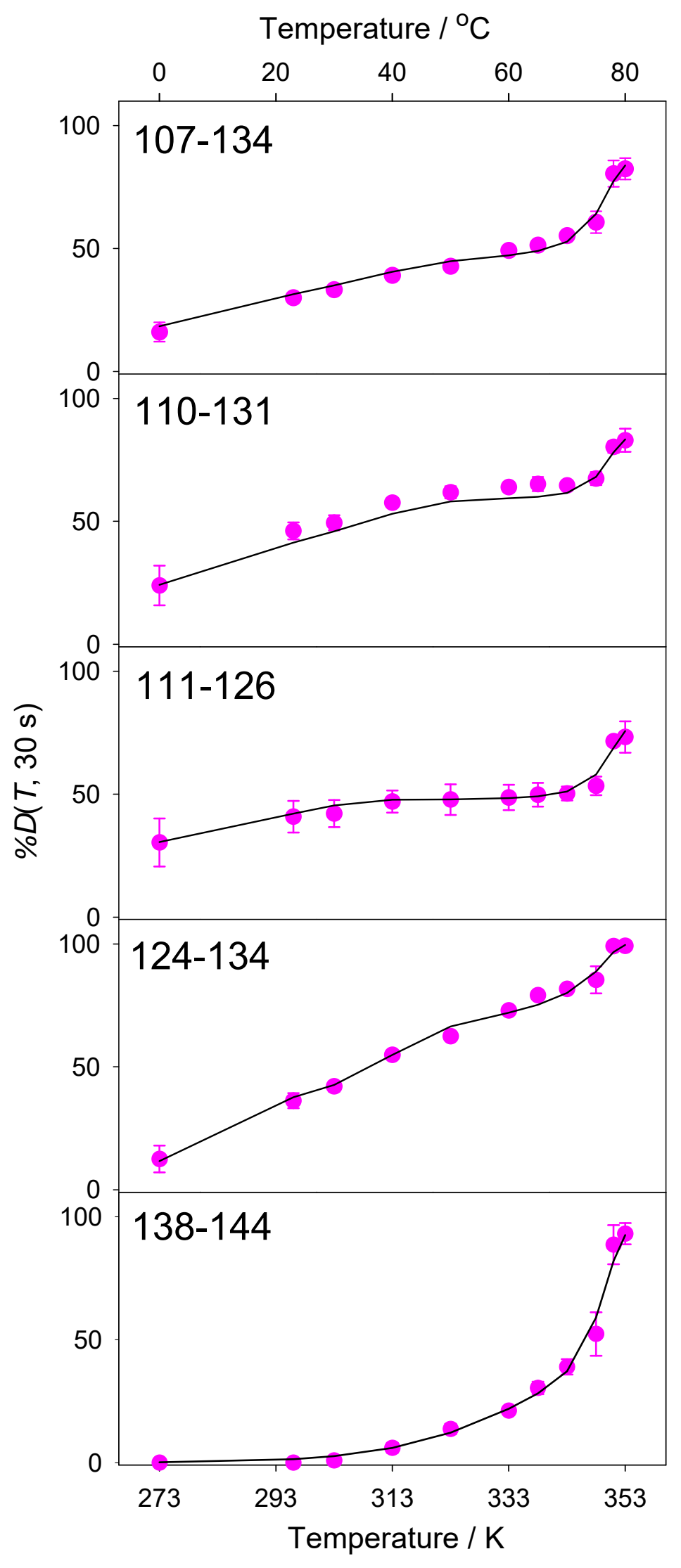

Time / s

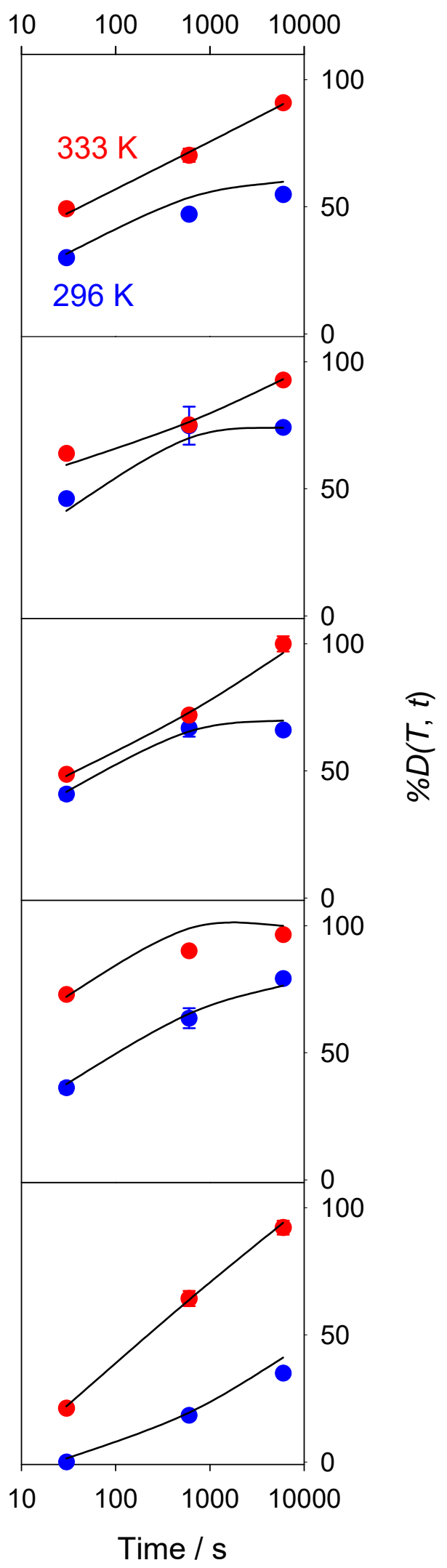




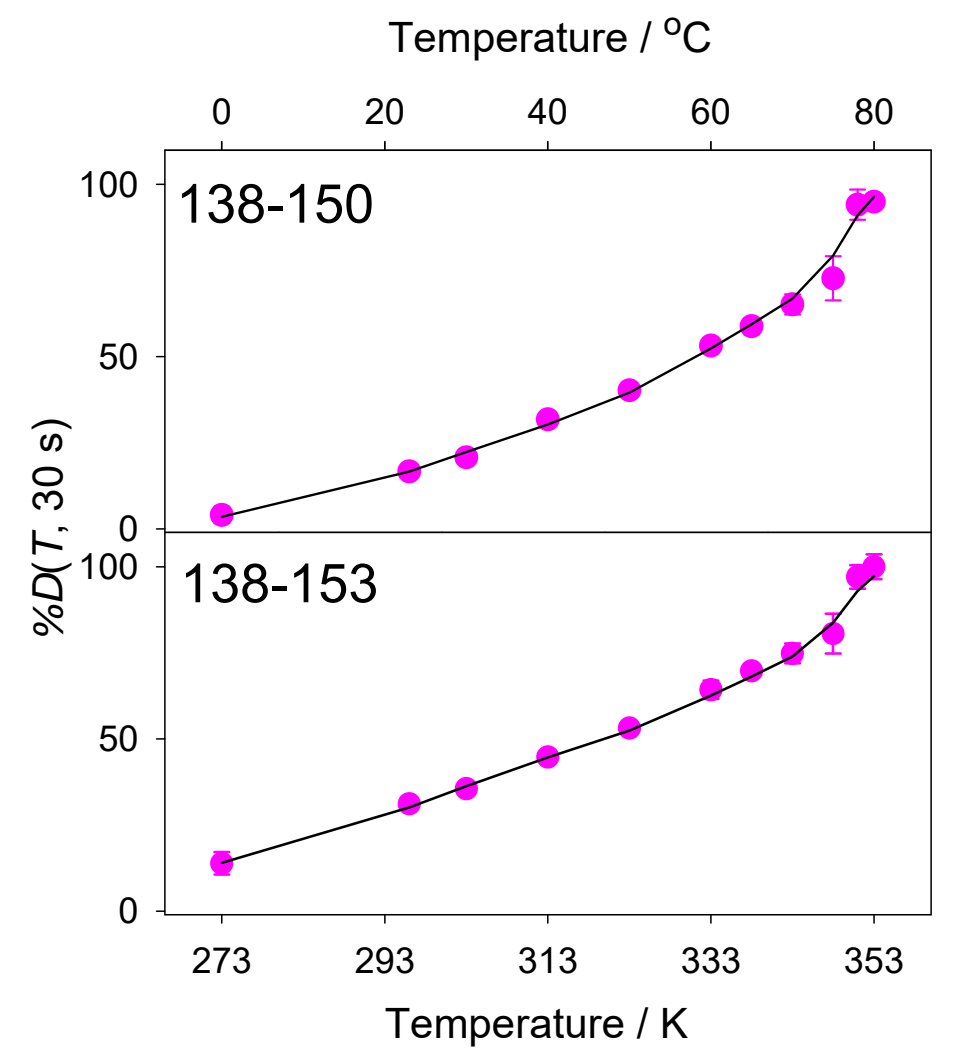

Time / s

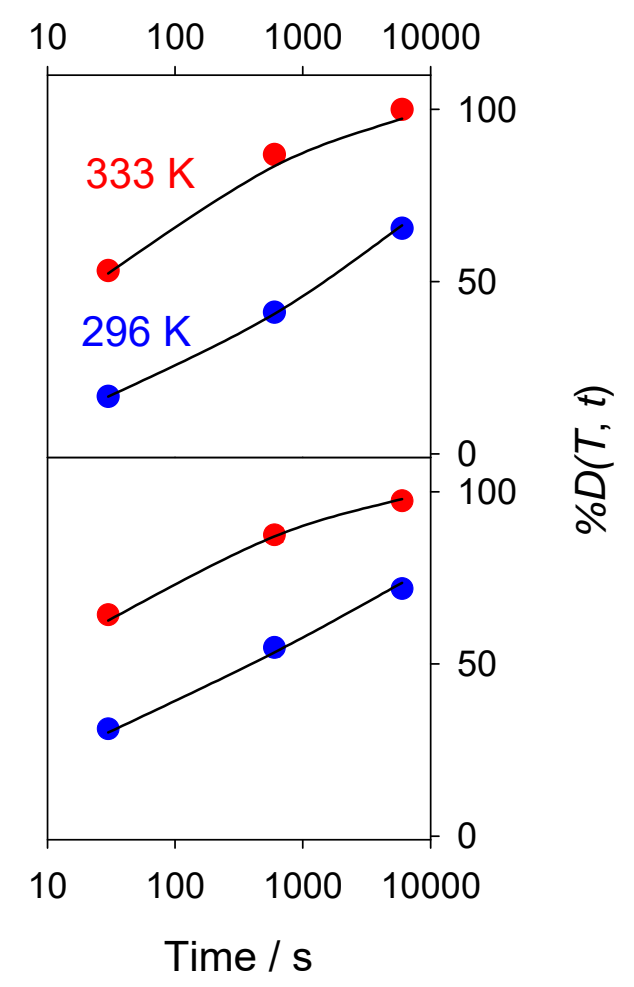




\section{SI References}

(1) Greenfield, N. J. Methods to Estimate the Conformation of Proteins and Polypeptides from Circular Dichroism Data. Anal. Biochem. 1996, 235, 1 - 10.

(2) Fersht, A. R. Structure and Mechanism in Protein Science; W. H. Freeman \& Co.: New York, 1999.

(3) Swint, L.; Robertson, A. D. Thermodynamics of unfolding for turkey ovomucoid third domain: Thermal and chemical denaturation. Protein Sci. 1993, 2, 2037-2049.

(4) Hamuro, Y. Determination of Equine Cytochrome c Backbone Amide Hydrogen/Deuterium Exchange Rates by Mass Spectrometry Using a Wider Time Window and Isotope Envelope. J. Am. Soc. Mass Spectrom. 2017, 28, 486-497.

(5) Kan, Z. Y.; Walters, B. T.; Mayne, L.; Englander, S. W. Protein hydrogen exchange at residue resolution by proteolytic fragmentation mass spectrometry analysis. Proc. Natl. Acad. Sci. U.S.A. 2013, 110, 16438-16443.

(6) Skinner, S. P.; Radou, G.; Tuma, R.; Houwing-Duistermaat, J. J.; Paci, E. Estimating Constraints for Protection Factors from HDX-MS Data. Biophys. J. 2019, 116, 1194-1203.

(7) Gessner, C.; Steinchen, W.; Bedard, S.; Skinner, J. J.; Woods, V. L.; Walsh, T. J.; Bange, G.; Pantazatos, D. P. Computational method allowing Hydrogen-Deuterium Exchange Mass Spectrometry at single amide Resolution. Sci. Rep. 2017, 7.

(8) Fajer, P. G.; Bou-Assaf, G. M.; Marshall, A. G. Improved Sequence Resolution by Global Analysis of Overlapped Peptides in Hydrogen/Deuterium Exchange Mass Spectrometry. $J$. Am. Soc. Mass Spectrom. 2012, 23, 1202-1208.

(9) Smith, D. L.; Deng, Y.; Zhang, Z. Probing the Noncovalent Structure of Proteins by Amide Hydrogen Exchange Mass Spectrometry. J. Mass Spectrom. 1997, 32, 135-146.

(10) Liang, Z.-X.; Lee, T.; Resing, K. A.; Ahn, N. G.; Klinman, J. P. Thermal-activated protein mobility and its correlation with catalysis in thermophilic alcohol dehydrogenase. Proc. Natl. Acad. Sci. U.S.A. 2004, 101, 9556-9561.

(11) Privalov, P. L.; Khechinashvili, N. N. A Thermodynamic Approach to the Problem of Stabilization of Globular Protein Structure: A Calorimetric Study. J. Mol. Biol. 1974, 86, 665-684.

(12) Konermann, L. In Encyclopedia of Life Sciences (eLS); John Wiley \& Sons, Ltd: Chichester, 2012.

(13) Nguyen, D.; Mayne, L.; Phillips, M. C.; Englander, S. W. Reference Parameters for Protein Hydrogen Exchange Rates. J. Am. Soc. Mass Spectrom. 2018, 29, 1936-1939.

(14) Reineke, K.; Mathys, A.; Knorr, D. Shift of pH-Value During Thermal Treatments in Buffer Solutions and Selected Foods. Int. J. Food Prop. 2011, 14, 870-881.

(15) Bai, Y.; Milne, J. S.; Mayne, L.; Englander, S. W. Primary Structure Effects on Peptide Group Hydrogen Exchange. Proteins: Struct., Funct., Genet. 1993, 17, 75-86.

(16) Covington, A. K.; Robinson, R. A.; Bates, R. G. The Ionization Constant of Deuterium Oxide from 5 to 50 Degrees. J. Phys. Chem. 1966, 70, 3820-3824.

(17) Yang, W. Y.; Gruebele, M. Folding at the speed limit. Nature 2003, 423, 193-197.

(18) Scalley, M. L.; Baker, D. Protein folding kinetics exhibit an Arrhenius temperature dependence when corrected for the temperature dependence of protein stability. Proc. Natl. Acad. Sci. U.S.A. 1997, 94, 10636-10640.

(19) Mallamace, F.; Corsaro, C.; Mallamace, D.; Vasi, S.; Vasi, C.; Baglioni, P.; Buldyrev, S. V.; Chen, S. H.; Stanley, H. E. Energy landscape in protein folding and unfolding. Proc. Natl. Acad. Sci. U.S.A. 2016, 113, 3159-3163. 